\title{
Contribution to Reactivity, Stability and Selectivity of Monodentated Free Phosphines
}

\author{
Kouadio Valery Bohoussou ${ }^{1}$, Anoubilé Bénié2, Mamadou Guy-Richard Koné ${ }^{1, \text { * }}$, \\ $\mathrm{N}^{\prime}$ guessan Yao Silvère Diki ${ }^{3}$, Koffi Alexis Respect Kouassi ${ }^{1}$, Nahossé Ziao ${ }^{1}$ \\ ${ }^{1}$ Faculty of Fundamental and Applied Sciences (UFR SFA), Laboratoire de Thermodynamique et de Physico-Chimie du Milieu, University \\ Nangui Abrogoua, Abidjan, Côte d'Ivoire \\ ${ }^{2}$ Faculty of Fundamental and Applied Sciences (UFR SFA), Laboratoire de Chimie Bio-Organique et de Substances Naturelles, University \\ Nangui Abrogoua, Abidjan, Côte d'Ivoire \\ ${ }^{3}$ Faculty of Science of Structures of Matter and Technology (UFR SSMT), Laboratoire de Chimie Physique, University Félix Houphouët - \\ Boigny, Abidjan-Cocody, Côte d'Ivoire
}

\author{
Email address: \\ guyrichardkone@gmail.com (M. Guy-Richard K.) \\ ${ }^{*}$ Corresponding author
}

\section{To cite this article:}

Kouadio Valery Bohoussou, Anoubilé Bénié, Mamadou Guy-Richard Koné, N'guessan Yao Silvère Diki, Koffi Alexis Respect Kouassi, Nahossé Ziao. Contribution to Reactivity, Stability and Selectivity of Monodentated Free Phosphines. Modern Chemistry.

Vol. 7, No. 2, 2019, pp. 38-44. doi: 10.11648/j.mc.20190702.13

Received: August 19, 2019; Accepted: September 2, 2019; Published: September 17, 2019

\begin{abstract}
Phosphines having a high degree of instability in their syntheses, it is necessary to find conditions for obtaining stable products in order to propose new stable and active compounds. It is this concern that responds to the theoretical study of stability and selectivity. This theoretical study of chemical reactivity was carried out using the Density Functional Theory (DFT) method, at the B3LYP/6-31G (p) calculation level. The use of the Frontier Molecular Orbital (FMO) theory, with the Single Orbital Molecular Orbital (SOMO) cation model, made it possible to study the stability of some isomers formed during the addition of free phosphines to the carbon-carbon triple bond. The analysis of these reactivity quantities allowed us to conclude that the presence of halogens on acetylene influences the stability of the stereoisomers during their formation. This stability increases as the electronegativity of the halogen decreases. The large number of $\pi$ conjugation favors the formation of Cis isomers and the lack or small amount of $\pi$ conjugation orients the formation of Trans isomers. The nature of the aryl substituent and the number of low electronegativity halogen on the phosphine are capable of promoting the production of stable products.
\end{abstract}

Keywords: DFT, Phosphine, Stability, SOMO, Stereoisomers

\section{Introduction}

Among the common approaches to controlling absolute stereochemistry, phosphine synthesis offers significant advantages. In fact, phosphines are very versatile precursors in synthesis [1]. They allow access to a range of molecules that are otherwise inaccessible or difficult to reach. These compounds have been used as starting materials in the fields of asymmetric catalysis [2], medicinal chemistry [3] and materials science with the realization of organic semiconductors [4]. They effectively catalyze innumerable reactions and have been essential to progress in cross- coupling and hydrogenation. Despite these diverse applications, there is still a limited amount of work on this compound family, in part because of its instability during its synthesis. With the development of computer techniques and computational chemistry, quantum chemistry gives insight into the electronic structures of molecules and propels the development of traditional experimental chemistry [5]. Currently, the method of functional density theory (DFT) has been accepted as a popular approach to calculate the structural characteristics and energies of molecules by the scientific community [6-9]. Its efficiency and accuracy in the evaluation of a number of molecular properties [10] have 
been recognized by all. Moreover, the examination and proper exploitation of frontier orbital energies (SOMO) guided the understanding of the chemical reactivity of free and complexed phosphines by Lee et al. by manipulating the electronic density as a fundamental quantity [11-13]. This work is part of the design and synthesis of new series of phosphine-based organic semiconductors. The objective of this work is to determine, by means of theoretical chemistry, the conditions for obtaining stable products during a hydrophosphination reaction.

\section{Material and Methods}

\subsection{Level of Theory}

Chemical species studies were performed using the theoretical Spartan 14 chemistry software [14]. The aim is to make a comparison with existing data in the literature. It allows the direct prediction of several molecular properties. The software gives access to several choices of methods such as Hartree-Fock, Post Hartree-Fock, DFT, semi-empirical. It is possible to carry out calculations in the gas phase or in the condensed state, in the ground state or in the excited state. It is a powerful tool for exploring the effects of substituents, the potential energy surface and excitation energies. Calculations were performed in vacuum, by the DFT method with the functional B3LYP $[15,16]$ with $6-31 \mathrm{G}$ (p) basis set. This Hybrid functional gives better energies and is in line with high-level ab initio methods [17, 18]. Consideration of the polarization functions is important especially because of the non-binding phosphorus pairs.

\subsection{Frontier Molecular Orbital (FMO) Theory}

Radical activation is a process often used for the hydrophosphination of single alkenes or alkynes. The formation reaction of free phosphines has been studied as a radical reaction. The mechanism involves the formation of a phosphoryl radical that adds to the unsaturated C-C bond [19, 20]. Thus, the reaction of a $\mathrm{P}-\mathrm{H}$ bond in the presence of a radical initiator and an olefin will lead to an addition on the double bond according to Figure 1 below:

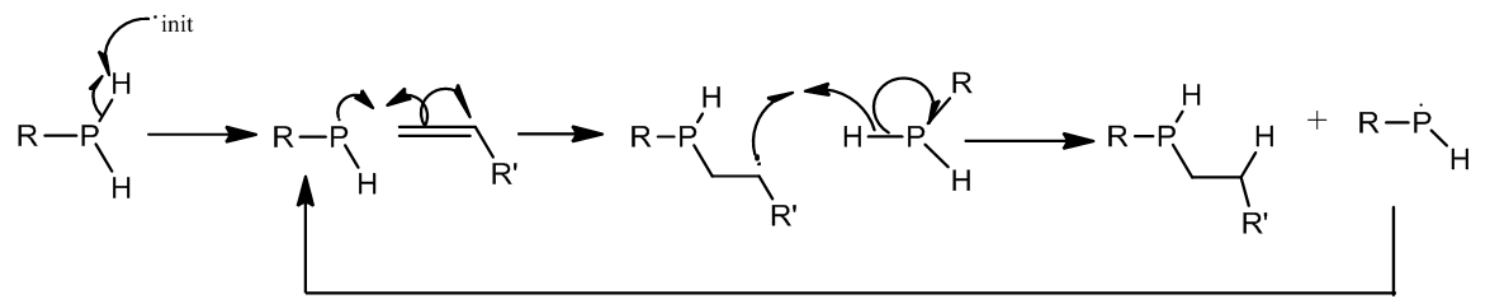

Figure 1. Mechanism of hydrophosphination initiated by radicals.

In radical reactions, the SOMO plays the role of a HOMO, or a LUMO, or both. SOMO energies have been specifically used by Lee Higham's group [21] to predict the reactivity of primary phosphines to oxygen in the air. This quantum parameter can foretell the reactivity of free phosphines with great accuracy.

\section{Results and Discussion}

\subsection{Theory of HOMO Frontier Orbitals in the Rationalization of the Reactivity of Free Phosphines}

According to Fukui's theory [22] of frontier orbitals, the electron density associated with HOMO electrons should explain reactivity and selectivity. In a family of molecules, the stability is even greater than this value is high. The energetic values of these descriptors for an addition of some free phosphines to dichloroethylene at B3LYP / 6-31G (p) level are shown in table 1 below.

Table 1. Energy of HOMO and LUMO orbitals of the studied phosphines.

\begin{tabular}{lllllll}
\hline & & & & \\
\end{tabular}


The values of the HOMO energies allow to establish the following sequence in order of increasing energy:

$$
\mathrm{E}_{\text {(номо) }}: 1 \mathrm{e}<1 \mathrm{f}<1 \mathrm{~b}<1 \mathrm{a}<1 \mathrm{~d}<1 \mathrm{c}<1 \mathrm{~g}
$$

According to the frontier orbitals theory, the compound $1 \mathrm{~g}$ which has the highest value of the HOMO energy is the most stable and the compound 1e is the least stable. Also, 1a is more stable than $2 \mathrm{~b}$. These results are in agreement with the experimental results published by Bénié et al. [23]. Indeed, according to these results, the compounds $1 \mathrm{a}$ and $1 \mathrm{~b}$ were less sensitive to the oxidation of the air than the compounds $1 \mathrm{~d}$ and 1c. This is not true on this classification scale. The HOMO and LUMO boundary orbitals do not allow to accurately rationalize the overall reactivity of free phosphines. In addition, the smaller the Gap (LUMO / HOMO), the more reactive the compound is $[24,25]$. Thus, the compound $1 \mathrm{~g}$ is more reactive and therefore less stable. These parameters therefore do not correctly reflect what has been obtained experimentally. We used the HOMO orbitals of SOMO cations according to the Lee et al model [21] for our study because of the match between theory and experiment.

\subsection{Lee Higham et al's Model}

A study of the stability of phosphines synthesized by Bénié et al. was performed at B3LYP / 6-31 (p) level. The values of the energies of the molecular orbitals SOMO obtained are represented by Figure 2 and Figure 3:

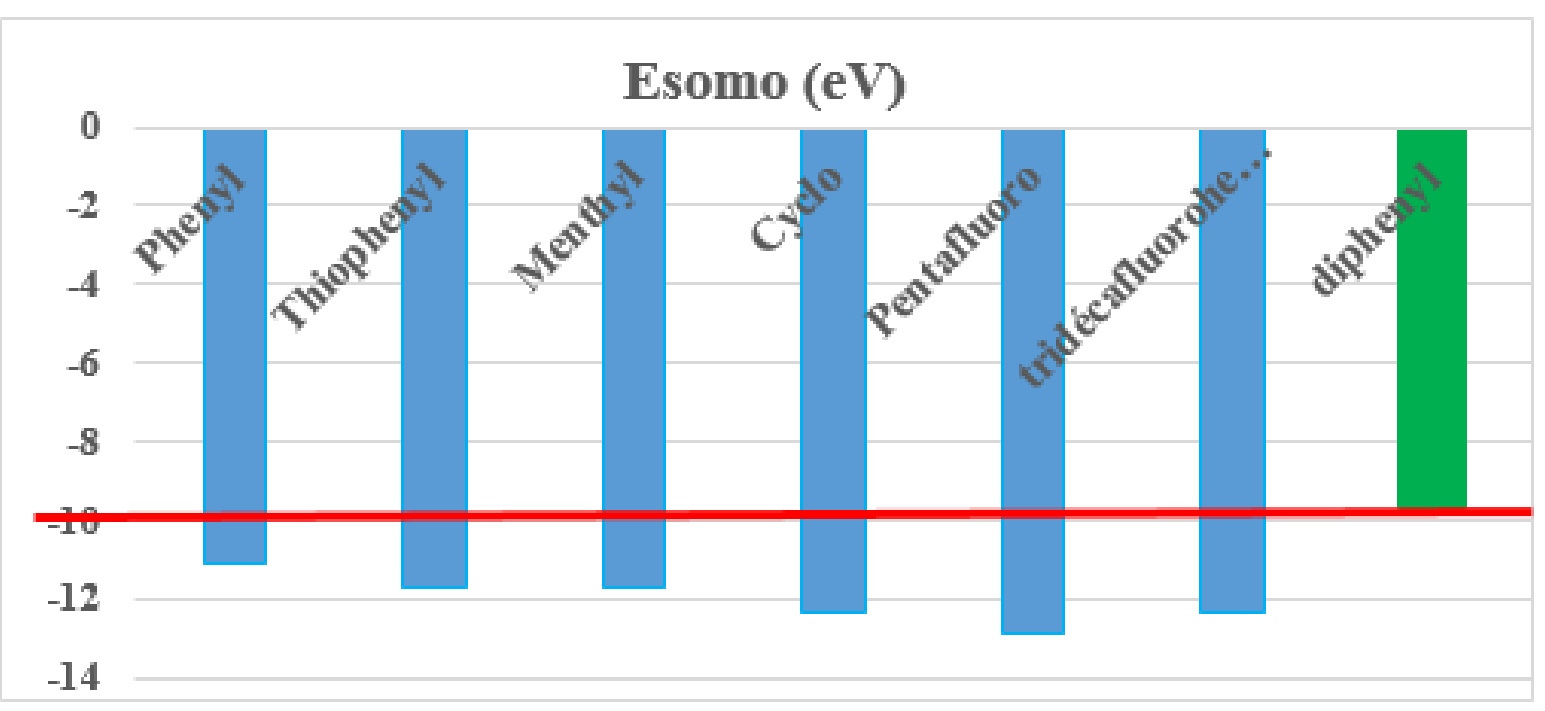

Figure 2. Histogram of the SOMO energies of the primary phosphines and the line threshold of stability in the air"at -10 $\mathrm{eV}$.

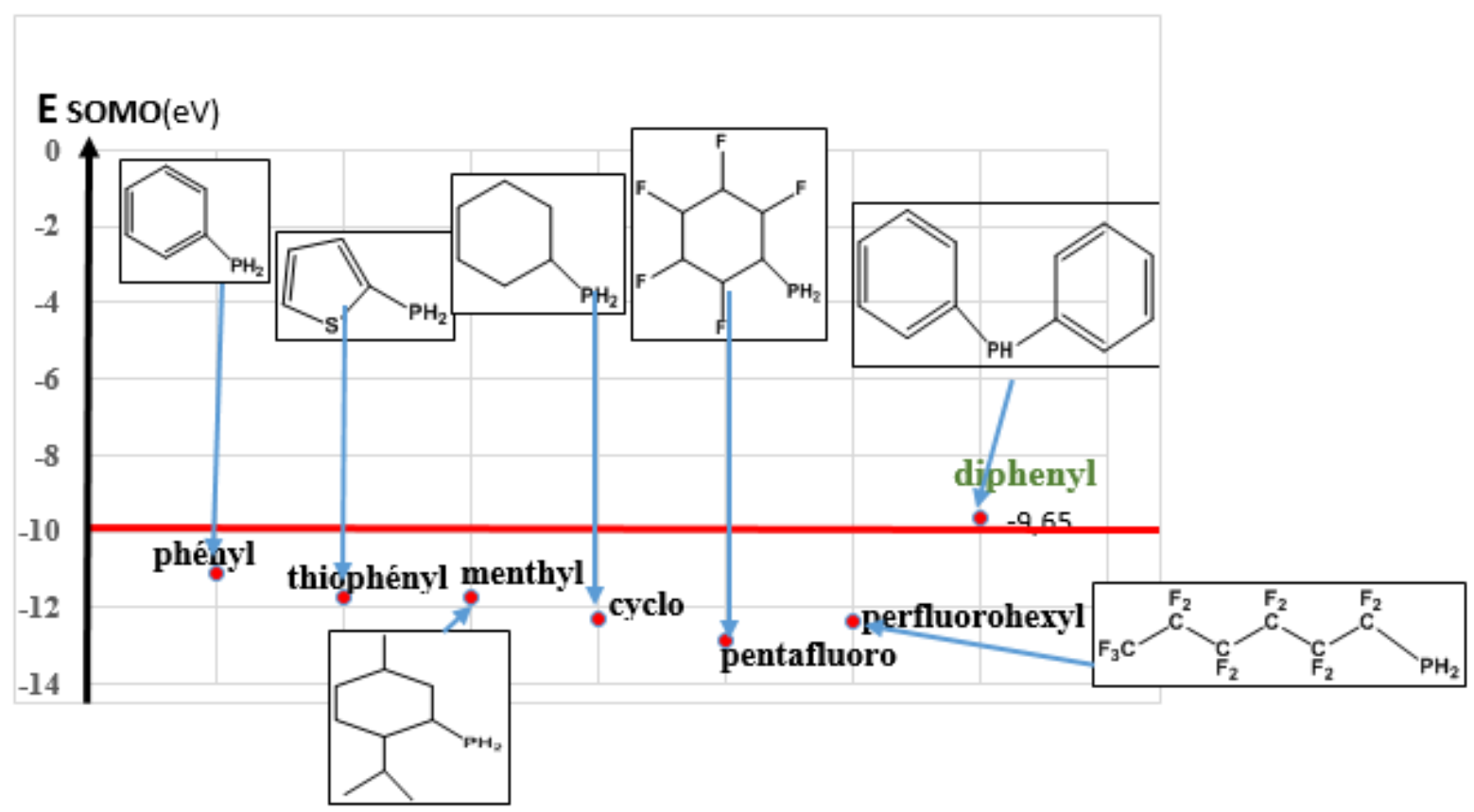

Figure 3. Graph showing the SOMO energies of the primary phosphines and the line Stability threshold in the air"at -10eV. 
The histogram reveals that diphenylphosphine $(2 \mathrm{~g})$ has the highest energy and pentafluoro Cyclohexylphosphine (2e) has the lowest energy SOMO. This energy exceeds the stability threshold proposed by Lee $\left(\mathrm{E}_{\text {somo }}>-10 \mathrm{eV}\right)$. The results of previous papers revealed that the stability of phosphines is highly dependent on heteroatoms, the possibility of delocalization of $\pi$ electrons in their structure and also their class. Thus, in the list examined, diphenylphosphine has a good stability due to its class and $\pi$ electrons which are in its structure. Diphenylphosphine is a secondary phosphine unlike perfluophosphine which is primary. The two aryl groups also increase its stability. This justifies the value of its energy $\left(\mathrm{E}_{\mathrm{SOMO}}>-10 \mathrm{eV}\right)$. This result is in perfect agreement with the experience.

\subsubsection{Influence of Halogen on the Stability of Phosphines}

A study of the stereoselectivity of some phosphines synthesized by Bénié et al. was conducted from Lee Higham's model. The values of the SOMO energies are given in Table 2 below. In this table, whatever the phosphine, we can establish the sequence of evolution of the SOMO values in descending order of energy for these three halogens studied as follows: ESOMO: $\mathrm{Br}>\mathrm{Cl}>\mathrm{F}$. This evolution follows the increasing order of the electronegativity of these halogens. Thus, the electronegativity of halogens influences the stability of the phosphines formed. Moreover, the results show that Cis isomers are more stable than their Trans counterparts. It is furthermore noted that only diphenylphosphine favors stable products with $\left(\mathrm{E}_{\mathrm{SOMO}}>\right.$ $10 \mathrm{eV})$. In addition, the stability of the products evolves in the same direction as that of the reagents.

Table 2. Values of SOMO energies of some free phosphines.

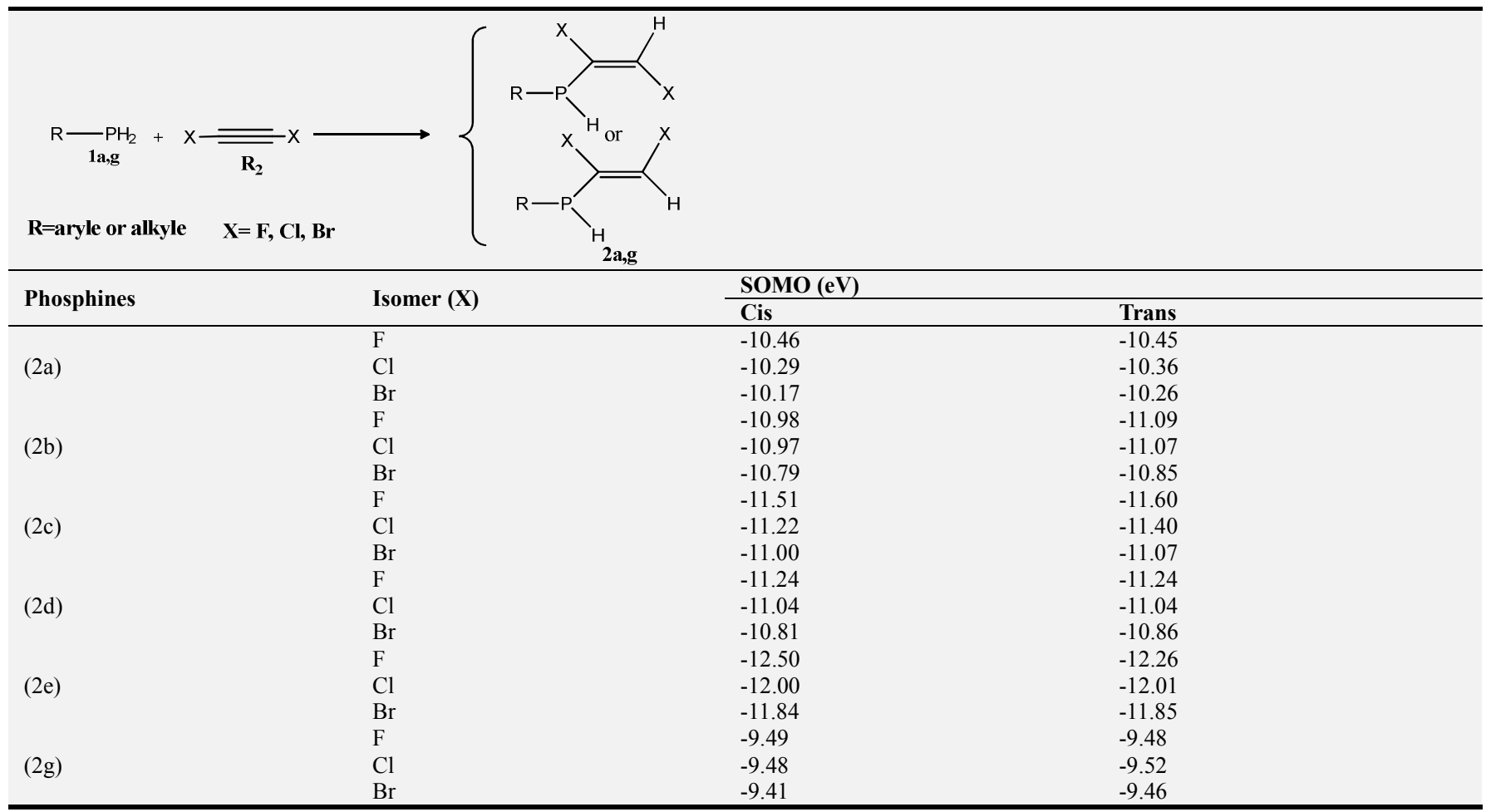

A study of the stability of some phosphines of the same class and having the same nucleus, but different only by the type of halogen was conducted from the model of Lee Higham. The values of the SOMO energies are gathered in Table 3 below: In this table, it is found that the SOMO values increase when less electronegative halogens are substituted for the phenyl. Thus, the stability of the free phosphines is a function of the electronegativity of the halogens used. It increases with decreasing electronegativity.

Table 3. SOMO energies values of some free phosphines as a function of halogens.

SOMO $(\mathrm{eV}) \mathrm{SHOSPHINES}$




\subsubsection{Influence of the Aryl Substituent on the Stability of Phosphines}

The results of the extension of the previous reaction with reagents and theoretical products are shown in Table 4.

Table 4. Value of SOMO energies of phosphines with aryl radicals.

\begin{tabular}{|c|c|c|c|}
\hline \multirow{2}{*}{$\begin{array}{l}\text { Substituent on } \\
\text { phosphorus }\end{array}$} & \multirow{2}{*}{ Isomers } & \multicolumn{2}{|c|}{ SOMO (eV) } \\
\hline & & Cis & Trans \\
\hline & $\mathrm{F}$ & -10.46 & -10.45 \\
\hline & $\mathrm{Cl}$ & -10.29 & -10.36 \\
\hline & $\mathrm{Br}$ & -10.17 & -10.26 \\
\hline & $\mathrm{F}$ & -10.38 & -10.39 \\
\hline (3b) & $\mathrm{Cl}$ & -10.26 & -10.37 \\
\hline & $\mathrm{Br}$ & -10.64 & -10.17 \\
\hline & $\mathrm{F}$ & -9.35 & -9.31 \\
\hline & $\mathrm{Cl}$ & -9.28 & -9.29 \\
\hline & $\mathrm{Br}$ & -9.20 & -9.24 \\
\hline & $\mathrm{F}$ & -9.56 & -9.67 \\
\hline & $\mathrm{Cl}$ & -9.54 & -9.56 \\
\hline & $\mathrm{Br}$ & -9.41 & -9.52 \\
\hline & $\mathrm{F}$ & -9.77 & -9.83 \\
\hline$(3 e)$ & $\mathrm{Cl}$ & -9.75 & -9.79 \\
\hline & $\mathrm{Br}$ & -9.67 & -9.76 \\
\hline
\end{tabular}

Analysis of the SOMO values reveals that vinylphenylphosphine "Trans" has a higher SOMO energy than the "Cis" form, when fluorine is used as halogen. In fact, for large values of electronegativities of halogens and for substituents having less space, the "Trans" form is more stable. On the other hand, "Cis" stereoisomers have a higher SOMO energy than their "Trans" counterparts for large aryl substituents and for halogens with low electronegativity values. The stereoisomers "Cis" are therefore more stable than the "Trans", when the size of the radicals on the phosphorus atom increases. The selectivity of the phosphines thus depends on the nature of the substituent on the phosphorus atom and the electronegativity of the halogens. Also, regardless of the stereoisomer "Cis" or "Trans", the stability of phosphines increases with the size of the radicals on the phosphorus atom. These results are in agreement with the experimental results of Brynda [1]. This stability also increases with the possibility of delocalization of the $\pi$ electrons. Which is in perfect agreement with the theoretical and experimental results of Lee's team [21].

\subsection{Comparison of the Global Stability of Free Phosphines}

\subsubsection{Influence of the Number of Halogen or Heteroatom}

A study of the global stability of some tertiary phosphines was conducted using Lee Higham's model. The values of SOMO energies are shown in Table 5 below:

Table 5. Value of SOMO energies of some tertiary phosphines.

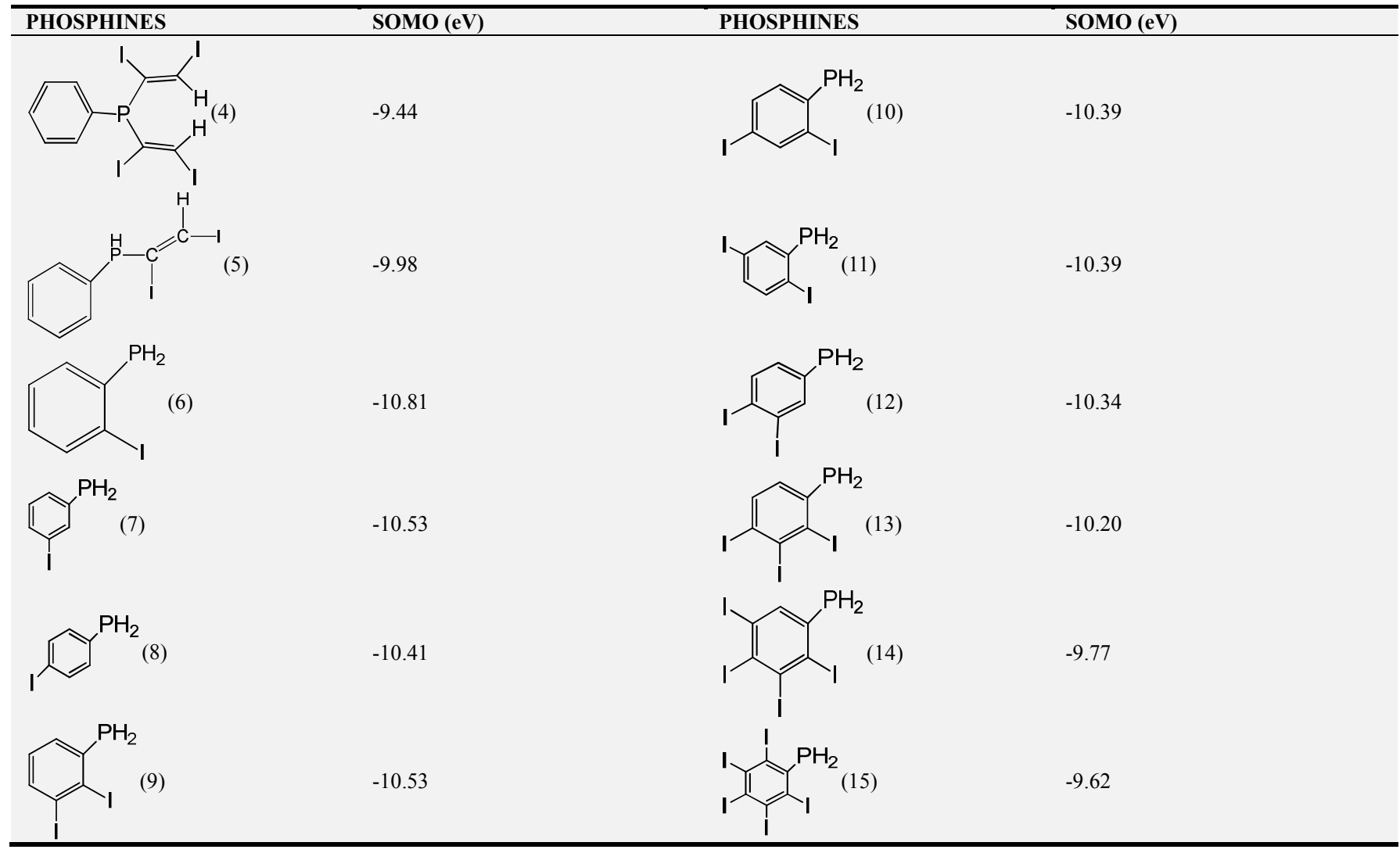


The results reveal that in this list, the compound (4) and (5) have a SOMO energy whose value is greater than the threshold $(-10 \mathrm{eV})$ proposed by Lee's model. The other compounds have a SOMO energy that is below the threshold. Thus, among these phosphines phosphine (4) is the most stable compound. Moreover, one can observe that the SOMO energies increase with the number of iodine atoms (halogen) on phenyl or on ethylene. But the increase of this energy is greater with ethylene substituted doubly with two hydrogen phosphorus. Double substitution promotes more congestion of phosphorus. The global stability of the free phosphines is related to the number of hetero atoms that the phosphine possesses. But also increases with the congestion of phosphorus.

\subsubsection{Influence of the Number of $\pi$ Conjugation}

A study of the global stability of some aryl and alkyl radical free phosphines was conducted using Lee Higham's model. The values of the energies of the molecular orbitals SOMO obtained are represented by Figures 4 and 5 .

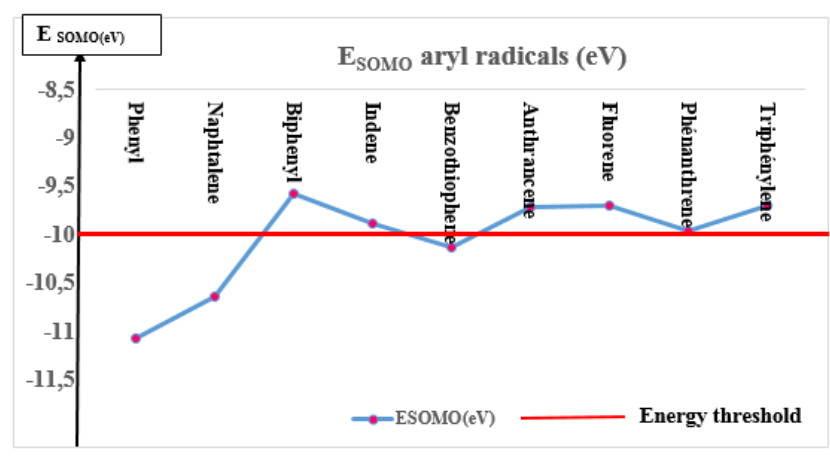

Figure 4. Representation of SOMO energies according to aryl radicals.

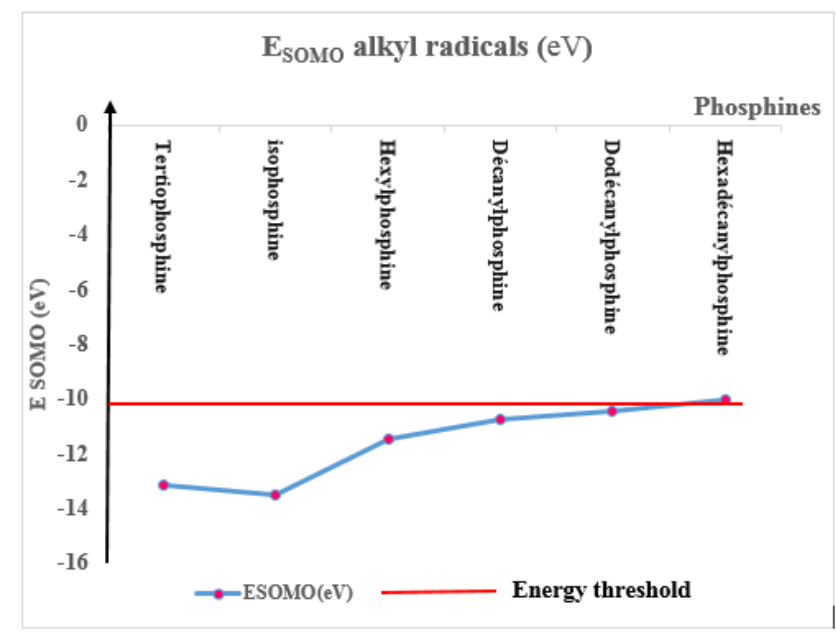

Figure 5. Representation of SOMO energies as a function of alkyl radicals.

These graphs reveal that phosphines which possess aryl radicals which favor a lot of $\pi$ electron delocalization give good stability according to the Lee criterion (ESOMO $\geq-10$ $\mathrm{eV}$ ). Alkylphosphines which do not have a SOMO energy of less than $-10 \mathrm{eV}$. It is also found that the energy SOMO increases as the size of the alkyl substituent increases $\mathrm{E}$ (Hexadecanylphosphine) $=-10 \mathrm{eV}$.

\subsubsection{Influence of the Halo-Aromatic Radical: Case of the Iodo-Aromatic}

A study of the global stability of some phosphines free of iodo-aromatic radicals was conducted from Lee Higham's model. The hydrogen atom was substituted with an iodine atom on the phenyl, naphthalene and indene group. The values of the energies of the molecular orbitals SOMO obtained are represented by Figure 6 .

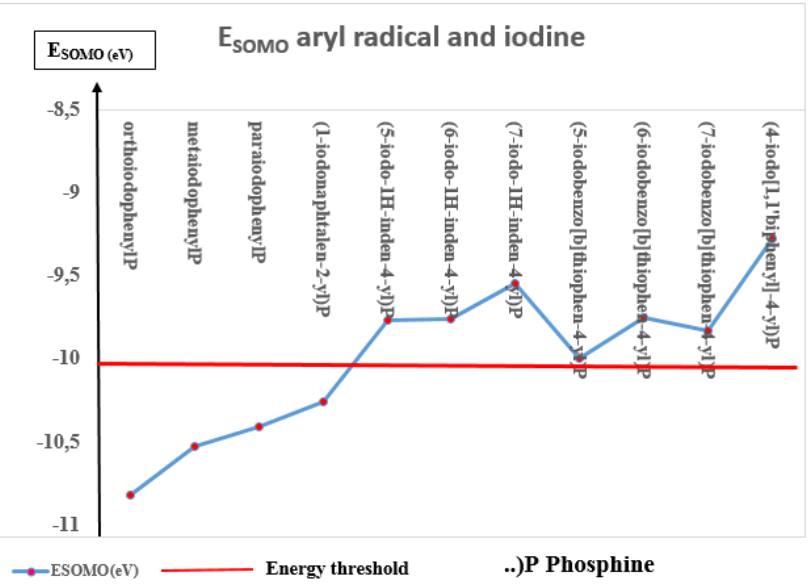

Figure 6. Representation of SOMO energies according to a combination of aryl radicals and iodine.

Examination of the graph reveals that the energy SOMO increases when the iodine is substituted for a hydrogen atom on phenyl, naphthalene, and on indene. This evolution of SOMO energy indicates that the substitution of iodine for aromatic radicals increases the stability of phosphines. Biphenylphosphine, for example, had its SOMO energy increase from -9.57 to $-9.28 \mathrm{eV}$. An adequate combination of halogens and aromatic radicals is a factor that increases the stability of free phosphines.

\section{Conclusion}

The use of the frontier molecular orbital (FMO) theory, with the model of the SOMO cation, made it possible to study the stability of some isomers formed during the addition of free phosphines to the carbon-carbon double and triple bonds. The analysis of these reactivity quantities allowed us to conclude that the presence of halogens on acetylene influences the stability of the stereoisomers during their formation. This stability increases as the electronegativity of the halogen decreases. The "Cis" form of vinylphosphines is more stable than the "Trans" form. The stability of free phosphines also increases when the substituent on phosphorus generates more $\pi$-conjugations on the aryl substituent. The nature of the aryl substituent and the number of low electronegativity halogen on the phosphine are capable of promoting the production of stable products. 


\section{References}

[1] M. Brynda, Coord. Chem. Rev., (2005) 249, 2013-2034.

[2] R. M. Hiney, A. Ficks, D. G. Gilheany, L. J. Higham, Airstablechiral primary phosphines part (i) synthesis, stability and applications, in: I. J. S. Fairlamb, J. M. Lynam (Eds.) Organometallic Chemistry: The Royal Society of Chemistry, London (2011) 37, 27-45.

[3] K. V. Katti, H. Gali, C. J. Smith, D. E. Berning, Acc. Chem. Res., (1998) 32, 9-17.

[4] H. Dorn, R. A. Singh, J. A. Massey, I. Manners, Angew. Chem., Int. Ed., (1999) 38, 3321-3323.

[5] J. B. Foresman, A. Frisch Exploring Chemistry with Electronic Structure Methods, Gaussian Inc., Pittsburgh, USA, (1996).

[6] M. Kurt, TR. Sertbakan, M. Ozduran. Spectrochim, An experimental and theoretical study of molecular structure and vibrational spectra of 3-and 4- pyridineboronic acid molecules by density functional theory calculations. Acta Part A: Mol. Biomol. Spectrosc. (2008); 70 (3): 664-673.

[7] L. H. A-Rahman, R. M. El-Khatib, L. A. E. Nassr, AM. ADief, M. Ismael, A. A. Seleem. Metal based pharmacologically active agents: Synthesis, structural characterization, molecular modeling, CT-DNA binding studies and in vitro antimicrobial screening of iron (II) bromosalicylidene amino acid chelates, Spectrochimica Acta Part A: Molecular and Biomolecular Spectroscopy (2014); $117 ; 366-378$.

[8] P. k Chattaraj, B. Maiti, U. Sarkar "philicity: a unified treatment of chemical reactivity and selectivity"j. Phys. Chem. $A$ (2003) 107 ; 25; 4973-4975.

[9] A. R. Jupp, T. C. Johnstone, D. W. Stephan. Improving the Global Electrophilicity Index (GEI) as a Measure of Lewis Acidity. Inorganic Chemistry (2018), 57 (23), 14764-14771.

[10] M. G. R. Koné, S. T. Affi, N. Ziao, K. Bamba, E. F. Assanvo. Hydrogen bonding sites in Benzimidazolyl-chalcones molecules: $\mathrm{Ab}$ initio and DFT investigation. Journal of Chemical and Pharmaceutical Research (2015); 7 (12): 805-812.

[11] Y. Traore, K. Bamba, N. Ziao, ST. Affi, M. G. R. Koné. Quantum chemical characterization of hydrogen bonding sites in three 4-(4-Halo-Phenyl)-6-(Furan-2-yl) Pyrimidin-2-Amine Derivatives, Computational Chemistry (2017); 5: 91-102.

[12] R. Bhattacharjee and R. K. Roy;"Relative Contribution of Combined Kinetic and Exchange Energy Terms vs the
Electronic Component of Molecular Electrostatic Potential in Hardness Potential Derivatives". The Journal of Physical Chemistry A (2013) 117 (45), 11528-11539.

[13] J. I. M. Araya, G. S. Morán, and D. G. Mitnik. Computational Nanochemistry Report on the Oxicams - Conceptual DFT Indices and Chemical Reactivity. The Journal of Physical Chemistry B (2013), 117 (21).

[14] Wavefunction Inc., Irvine, CA, (2014).

[15] Frisch MJ, Trucks GW, Schlegel HB, Scuseria GE. Gaussian 09, Revision A.02. Gaussian, Inc., Wallingford CT; (2009).

[16] Lee C, Yang W, Parr RG. Development of the Colle-Salvetti correlation-energy formula into a functional of the electron density. Phys. Rev. (1988) B37 (2): 785-789.

[17] J. Kapp, M. Remko, PVR. Schleyer. $\mathrm{H}_{2} \mathrm{XO}$ and $\left(\mathrm{CH}_{3}\right)_{2} \mathrm{XO}$ Compounds $(\mathrm{X}=\mathrm{C}, \mathrm{Si}, \mathrm{Ge}, \mathrm{Sn}, \mathrm{Pb})$ : Double bonds vs carbenelike structures can the metal compounds exist at all? Journal of the American Chemical Society. (1996) 118: 5745-5751.

[18] AD. Becke. Density-functional thermochemistry. III. The role of exact exchange. J. Chem. Phys. (1993) 98 (7): 5648-5652.

[19] L Maier. Helv. Chim. Acta (1966), 842-851.

[20] J. C. Guillemin; S. L. Serre; L Lassalle. Adv. Space Res. (1997) 19, 1093-1102.

[21] B. Stewart, H. Anthony, and H. J. Lee Predicting the Air Stability of Phosphines American Chemical Society Organometallics (2011) 30, 5338-5343.

[22] F. H. Zadeh, R. A. M. Quintana, T. Verstraelen, P. Bultinck, and P. W. Ayers;" When is the Fukui Function Not Normalized? The Danger of Inconsistent Energy Interpolation Models in Density Functional Theory". Journal of Chemical Theory and Computation (2016), 12 (12), 5777-5787.

[23] A. Benié, J. M. Dénis, Y. A Békro, Synthesis of Free and Complexes 2,2 Dichloro (ethyl) Aryl phosphine and bis(2,2)Dichloro(ethyl)Arylphosphine by Regioselective Hydrophosphination. European Journal of Scientific Research (2010) 47, 164- 168.

[24] K. N. N'Guessan, K. Bamba, O. P. Wawohinlin, N. Ziao; NBO Population Analysis and Electronic Calculation of Four Azopyridine Ruthenium Complexes by DFT Method; Computational Chemistry (2017), 5, 51-64.

[25] R. K. Singh, S. K. Verma, and P. D. Sharma, DFT Based Study of Interaction between Frontier Orbitals of Transition Metal Halides and Thioamides. International Journal of Chemtech Research, (2011) 3, 1571-1579. 